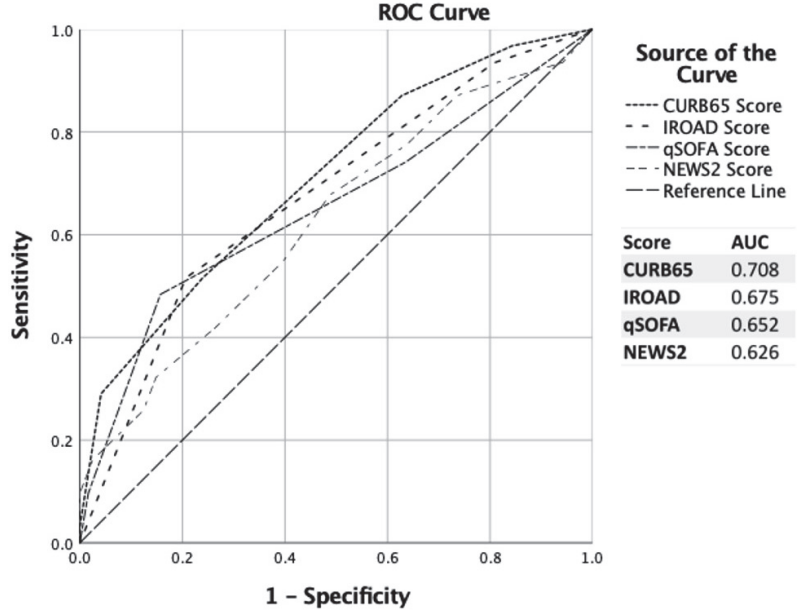

Abstract S16 Figure 1 Receiver operating characteristic (ROC) curves and area under the curve (AUC) values for 30-day mortality prediction by CURB65, IROAD, qSOFA and NEWS2 scoring systems

univariate analysis. The accuracy of the scoring systems in predicting 30-day mortality was assessed using receiver operating characteristic (ROC) analysis.

Results We identified 323 patients with an ICD-10 coded diagnosis of NV-HAP. 154/323 (48\%) met the diagnostic criteria for inclusion. 30-day mortality in these included patients was $21.4 \%$. Univariate analysis revealed advanced age, emergency admission, speech/swallowing difficulties, altered mental status, lymphocyte count $<0.7 \times 10^{9} / \mathrm{L}$, neutrophil-to-lymphocyte-ratio $\geq 10$, urea $>7 \mathrm{mmol} / \mathrm{L}$, acute kidney injury, CURB65 $\geq 2, \mathrm{qSOFA} \geq 2$ and $\mathrm{IROAD}=$ 'severe' as being significantly associated with 30-day mortality. Raised white blood cell count, C-reactive protein, the extent of radiographic features, prior antibiotic therapy and NEWS $2>4$ were not significantly associated with mortality. In ROC analysis, scoring systems achieved the following area under the curve values: CURB65-0.708; IROAD-0.675; qSOFA-0.652 and NEWS2-0.626 (figure 1).

Conclusion NV-HAP-specific scoring systems are needed, as current scoring systems have limited prognostic accuracy. Larger studies with the power to perform multivariate analysis are required to validate the factors identified as being associated with mortality. These factors may be used to create a more accurate, NV-HAP-specific scoring system.

\section{S17 DYNAMIC EARLY WARNING SCORE VERSUS NATIONAL EARLY WARNING SCORE-2 FOR PREDICTING CLINICAL DETERIORATION IN RESPIRATORY PATIENTS}

${ }^{1} \mathrm{~S}$ Gonem, ${ }^{2} \mathrm{~A}$ Taylor, ${ }^{2} \mathrm{G}$ Figueredo, ${ }^{2} \mathrm{~S}$ Forster, ${ }^{2} \mathrm{~T}$ McKeever, ${ }^{2} \mathrm{~S}$ Garibaldi, ${ }^{2} \mathrm{D}$ Shaw. ${ }^{1}$ Nottingham University Hospitals NHS Trust, Nottingham, UK; ${ }^{2}$ University of Nottingham, Nottingham, UK

\subsection{6/thorax-2021-BTSabstracts.23}

Background The National Early Warning Score-2 (NEWS2) is used to detect patient deterioration in UK hospitals but relies on a snapshot assessment. We developed a Dynamic Early Warning Score (DEWS) which takes into account changes over time, and assessed its accuracy in respiratory patients for predicting death or intensive care unit (ICU) admission, both within 24 hours, and clinical deterioration within 4 hours.
Methods Clinical observations data were extracted from electronic data records for 31590 respiratory in-patient episodes (53.5\% female, mean age 69.9) at a large acute NHS Trust from $1^{\text {st }}$ April 2015 to $31^{\text {st }}$ December 2020, comprising 1037349 date and time-stamped observation sets. 786 inpatient episodes comprising 52300 observation sets were annotated manually following reference to the medical case-notes. Clinical deterioration was defined as a specific event (eg. hospital-acquired pneumonia) requiring a change in treatment (eg. antibiotics). Development of DEWS used similar methodology to Zhu et al (Resuscitation. 2020; 157: 176-84). Continuous variables were divided into two separate variables corresponding to high or low values. Time series features including rolling average, standard deviation and trend over previous observations were entered into a logistic regression model. For death or ICU admission within 24 hours, data from 2015-2019 and 2020 were used for model training and validation respectively. For clinical deterioration within 4 hours, Results presented are for the training dataset (validation dataset is pending).

Results Death or ICU admission occurred within 24 hours of $2.3 \%$ of observations sets. The area under the receiver operating curve (AUC $[95 \%$ confidence interval]) for predicting death or ICU admission within 24 hours was 0.903 (0.897 $0.911)$ for DEWS versus $0.862(0.859-0.865)$ for NEWS2 in the training dataset, and $0.901(0.892-0.908)$ for DEWS versus $0.854(0.849-0.858)$ for NEWS2 in the validation dataset. Clinical deterioration occurred within 4 hours of $6.6 \%$ of observation sets. The AUC for predicting clinical deterioration within 4 hours was $0.861(0.842-0.878)$ for DEWS versus $0.793(0.783$ - 0.801) for NEWS2.

Conclusions DEWS has superior performance compared to NEWS2 with respect to predicting death or ICU admission within 24 hours, and clinical deterioration within 4 hours, in respiratory patients.

Please refer to page A188 for declarations of interest related to this abstract.

\section{S18 INVESTIGATING THE IMPACT OF INFLUENZA ACTIVITY ON EXCESS MORTALITY RATES FROM CARDIOVASCULAR, RESPIRATORY AND RENAL DISEASES IN IRELAND DURING THE 2010/11-2019/20 INFLUENZA SEASONS}

${ }^{1,2} \mathrm{JX}$ Choo, ${ }^{2,3} \mathrm{~J}$ Harbison. 'School of Medicine, Trinity College Dublin, Dublin, Ireland; ${ }^{2}$ Mercer's Institute for Successful Ageing, St James's Hospital, Dublin, Ireland; ${ }^{3}$ Department of Medical Gerontology, School of Medicine, Trinity College Dublin, Dublin, Ireland

10.1136/thorax-2021-BTSabstracts.24

Introduction COVID-19 and influenza infection are associated with cardiovascular, respiratory and renal complications. However, cardiovascular, respiratory and renal disease mortality rates in Ireland decreased by $0.04 \%, 0.12 \%$ and $0.12 \%$ respectively in 2020 relative to 2019 , despite nearly 100,000 new COVID-19 cases being reported in Ireland in 2020. Government-imposed social distancing measures resulted in abolition of influenza activity (IA). We analysed population data from the 2010/11-2019/20 influenza seasons to estimate the impact of reduced IA on cardiovascular, respiratory and renal disease mortality rates during the COVID-19 pandemic season. Methods Quarterly mortality data for acute myocardial infarction (AMI), cerebrovascular disease, pneumonia, asthma and renal disease from first quarter (Q1) 2010 to fourth quarter (Q4) 2020 was obtained from the Central Statistics Office. 
Weekly data on influenza-like illness (ILI) rates and positive percentages (PP) (i.e., proportion of influenza-positive sentinel respiratory specimens) was obtained from the Health Protection Surveillance Centre. Excess mortality rate during influenza season was defined as the percentage difference between Q4/ Q1 and preceding third quarter (Q3) mortality rates. We adopted the Goldstein Index (ILI rate $\times$ PP) as an indicator of IA. Time series analyses, Pearson correlation coefficients (r) and linear regression models were used to evaluate the relationships between IA and excess cardiovascular, respiratory and renal disease mortality rates.

Results Statistically significant positive associations were observed between IA and excess AMI $(r=0.557, p=0.011)$, cerebrovascular disease $(\mathrm{r}=0.858, \mathrm{p}<0.001)$, pneumonia $(\mathrm{r}=0.635, \mathrm{p}=0.003)$, asthma $(\mathrm{r}=0.668, \mathrm{p}=0.001)$ and renal disease $(\mathrm{r}=0.652, \mathrm{p}=0.002)$ mortality rates. Linear regression models predicted $0.072 \%$ (95\% confidence interval $0.019 \%$, $0.125 \%), \quad 0.095 \% \quad(0.067 \%, \quad 0.123 \%), \quad 0.184 \% \quad(0.073 \%$, $0.296 \%), 0.367 \%(0.165 \%, 0.569 \%)$ and $0.124 \%(0.053 \%$, $0.196 \%$ ) increases in excess AMI, cerebrovascular disease, pneumonia, asthma and renal disease mortality rates respectively per unit increase in IA.

Conclusion Elimination of IA may have contributed towards limiting the effects of COVID-19 on cardiovascular, respiratory and renal disease mortality rates in Ireland.

\section{S19 RELEVANCE OF PREDICTION SCORES DERIVED FROM THE SARS-COV-2 FIRST WAVE, IN THE UK COVID-19 SECOND WAVE, FOR EARLY DISCHARGE, SEVERITY AND MORTALITY: A PREDICT COVID UK PROSPECTIVE OBSERVATIONAL COHORT STUDY}

H Ghani, A Navarra, E Croft, H Nur, M Prabhakar, A Azri Yahaya, I Darwish, D Longe, HL Lee, R Vancheeswaran. West Hertfordshire Hospitals NHS Trust, Watford, UK
Introduction and Objective COVID-19 prognostication scores are all based on COVID-19 first wave, requiring prospective validation in the evolving pandemic due to SARS-CoV-2 variants (prevalent B.1.1.7 replacing parent D614) and healthcare responses altering patient demographic and mortality. Accelerated COVID-19 virtual hospital (VH) telemedicine model implementation avoids hospital admission, appropriately allocating hospital resources to pandemic needs in tandem with resumption of regular healthcare services, requires a safe triage tool. Widely used COVID-19 first wave derived prognostication scores, SOARS and 4C Mortality Score, with uncertain performance in the evolving pandemic, raises the need for prospective validation. We prospectively validate SOARS and 4C Mortality Score in the evolving UK COVID-19 second wave determining relevance for mortality and safe early discharge.

Methods Protocol-based, prospective observational cohort study of SOARS and 4C Mortality Score in 1,383 PREDICT (single site) and 20,595 multi-site ISARIC (International Severe Acute Respiratory and Emerging Infections Consortium) patient cohorts during the UK COVID-19 second wave, between October 2020 and January 2021

Results Data from 1,383 patients (median age 67y, IQR 5282; mortality 24.7\%) in the PREDICT and 20,595 patients from the ISARIC (mortality 19.4\%) cohorts showed SOARS had AUC of 0.8 and 0.74 , while 4C Mortality Score had an AUC of 0.83 and 0.91 for hospital mortality, in the PREDICT and ISARIC cohorts respectively, therefore effective in evaluating both safe discharge and in-hospital mortality. 19.3\% (231/ 1195, PREDICT cohort) and 16.7\% (2550/14992, ISARIC cohort) with a SOARS of $0-1$ were potential candidates for home discharge to a virtual hospital (VH) model. SOARS score implementation resulted in low re-admission rates, $11.8 \%(27 / 229)$, and low mortality, $0.9 \%(2 / 229)$, in the $\mathrm{VH}$ pathway. Use is still suboptimal to prevent admission, as $8.1 \%$

10.1136/thorax-2021-BTSabstracts.25

\section{SOARS and 4C Mortality Score in PREDICT and ISARIC Cohorts}
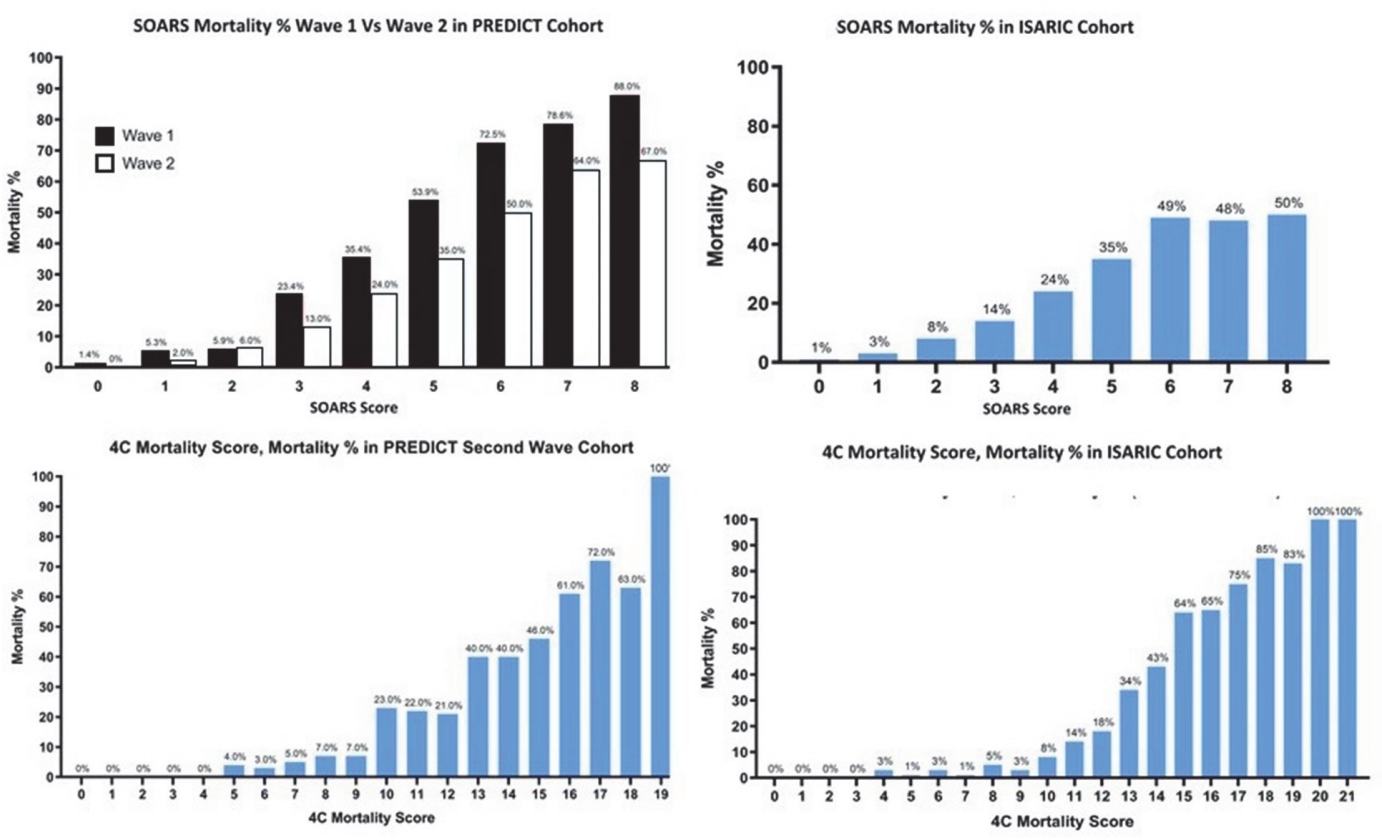

Abstract S19 Figure 1 SOARS and 4C mortality score in PREDICT and ISARIC cohorts 